\title{
ChemComm
}

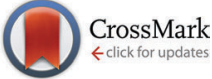

Cite this: Chem. Commun., 2016, 52,2768

Received 23rd December 2015, Accepted 5th January 2016

DOI: $10.1039 / \mathrm{c} 5 \mathrm{cc} 10527 \mathrm{~g}$

www.rsc.org/chemcomm

\section{Attraction by repulsion: compounds with like charges undergo self-assembly in water that improves in high salt and persists in real biological fluids $\uparrow$}

\author{
Graham A. E. Garnett, ${ }^{a}$ Kevin D. Daze, ${ }^{a}$ Jorge A. Peña Diaz, ${ }^{a}$ Noah Fagen, ${ }^{a}$ \\ Alok Shaurya, ${ }^{a}$ Manuel C. F. Ma, ${ }^{a}$ Mary S. Collins, ${ }^{b}$ Darren W. Johnson, \\ Lev N. Zakharov ${ }^{c}$ and Fraser Hof*a
}

\begin{abstract}
We report a family of highly anionic calixarenes that form discrete homo-dimeric assemblies in pure water, that get stronger in high salt solutions, and that remain assembled in complex, denaturing solutions like real urine. The results reveal the potential of like-charged subunits for self-assembly in high-salt solutions and biological fluids.
\end{abstract}

Most successes in 'bio-supramolecular chemistry' that function in pure water have come from agents that combine rigid, concave structures, a hydrophobic component, and mutual electrostatic attraction between multiple cationic and anionic groups on either partner. Using mutual electrostatic attraction reliably promotes assembly in organic solvents, but has a mixed record of success upon transfer of discrete assemblies to pure water and/or salty, buffered solutions. ${ }^{1-6}$ Regardless of the successes or failures of such designs, it is a general feature that the addition of salts (like those found in all biological fluids) will weaken electrostatically driven complexes by increasing the dielectric ${ }^{6}$ and/or directly competing for binding sites. ${ }^{7}$ Salts also weaken ion binding by neutral hosts like cucurbiturils. ${ }^{8}$ Denaturing organic co-solutes (such as urea in urine) create a further challenge for synthetic molecular recognition systems intended to work in real biological fluids. We report here a new assembly motif in which similarly charged building blocks bind to each other faithfully in water, form a stronger complex upon addition of salt, and remain assembled in complex solutions like mock serum and real urine. Our results show the unexpected advantages of using like-charged molecular recognition elements in high-salt solutions.

\footnotetext{
${ }^{a}$ Department of Chemistry, University of Victoria, PO Box 3065, STN CSC, Victoria, v8W3V6, Canada. E-mail: fhof@uvic.ca

${ }^{b}$ Department of Chemistry \& Biochemistry, University of Oregon, Eugene, Oregon, 97403-1253, USA

'Center for Advanced Materials Characterization in Oregon (CAMCOR),

1241 University of Oregon, Eugene, OR 97403-1241, USA

$\dagger$ Electronic supplementary information (ESI) available: Synthesis, characterization of new compounds, and supplemental data for characterization of assemblies by NMR, ITC, and X-ray crystallography. CCDC 1033560 . For ESI and crystallographic data in CIF or other electronic format see DOI: 10.1039/c5cc10527g
}

We previously prepared mono-functionalized, sulfonated calixarenes because the appended group tunes the recognition of methyllysine guests within the binding pocket. ${ }^{9,10}$ In the course of this work, we noticed that tolyl-substituted compound 2 (Fig. 1) has an unusual NMR spectrum in water. Resonances for the pendant methyl group are shifted upfield by up to $2.8 \mathrm{ppm}$ in $\mathrm{D}_{2} \mathrm{O}$ and in phosphate-buffered $\mathrm{D}_{2} \mathrm{O}$, suggesting aggregation. The resonances return to their normal chemical shifts upon dilution (Fig. 2a), showing that intermolecular association is responsible for the observed shifts. No signs of assembly exist in $\mathrm{CD}_{3} \mathrm{OD}$ (Fig. 2a).

We prepared analogs 1-5 bearing a series of alkyl substituents in order to characterize this mode of assembly. Each analog forms assemblies similar to those of the parent. Upfield chemical shifts occur in $\mathrm{D}_{2} \mathrm{O}$ (Fig. 1b) and in phosphate-buffered $\mathrm{D}_{2} \mathrm{O}$, but not $\mathrm{CD}_{3} \mathrm{OD}(\mathrm{ESI} \dagger)$. The shifts are concentration dependent and return to normal values upon dilution (ESI $\dagger$ ). The chemical shift perturbations show that the pendant arm in each compound is bound in a calixarene pocket in an end-on manner (Fig. 1b). Molecular modeling suggests this can only be achieved in a homodimer arrangement, and diffusion constants $(D)$ obtained for 5 by DOSY-NMR (Table 1) and NOE data (Fig. 1c, ESI $\dagger$ ) further support formation of a homodimer. The single crystal X-ray structure of 5, obtained after slow evaporation of water, revealed a homodimeric structure that is consistent with all solution-phase NMR data (Fig. 1d). $\$$

The thermodynamics of dimer formation reveal the influence of charge and salt on the assemblies. Isothermal titration calorimetry (ITC) dilution titrations carried out in the same phosphate buffer as NMR experiments show low-mM $K_{\mathrm{d}}$ values (Table 2). The strength of binding increases with increasing hydrophobic surface area. The same ITC experiments were repeated in phosphate buffered saline containing the concentrations of $\mathrm{NaCl}, \mathrm{KCl}$, and $\mathrm{MgCl}_{2}$ found in human serum (Table 2). Each of 1-5 assembled more strongly in the high-salt solution, with the strongest dimer $\mathbf{5}$ reaching a $K_{\mathrm{d}}$ of $730 \mu \mathrm{M}$ under these conditions.

The very low $\mathrm{p} K_{\mathrm{a}}$ 's of sulfonic acids $\left(\mathrm{p} K_{\mathrm{a}}<0\right)$ and of the first phenol OH in a calix[4] arene scaffold $\left(\mathrm{p} K_{\mathrm{a}}=3.26\right)^{11}$ mean that each monomer 1-5 is tetra-anionic under the buffered conditions 

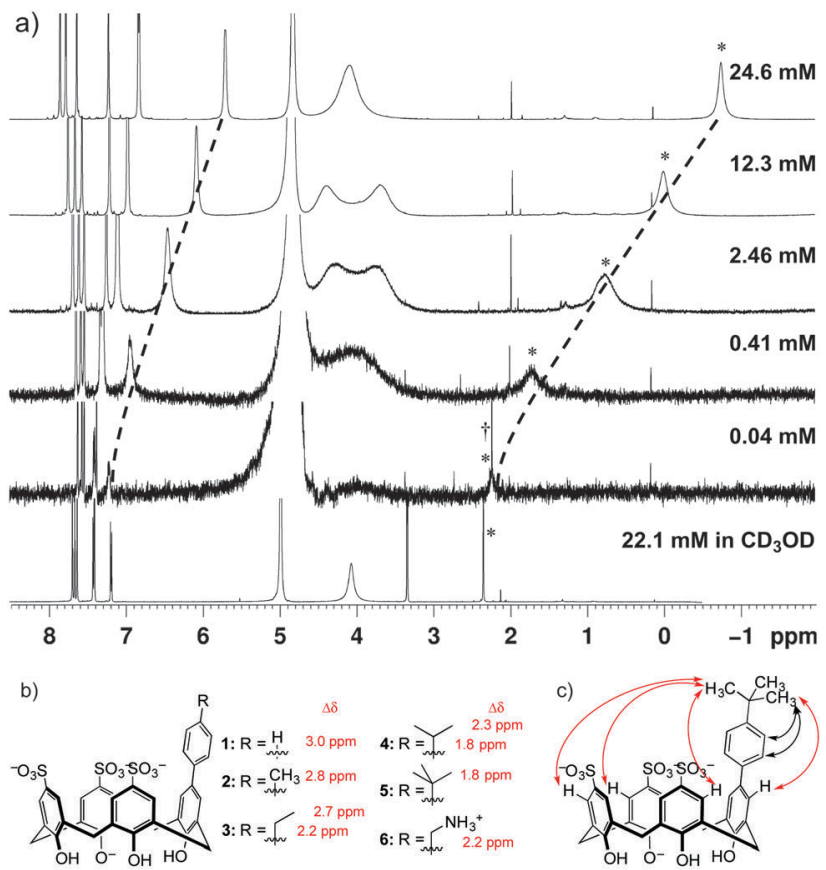

d)
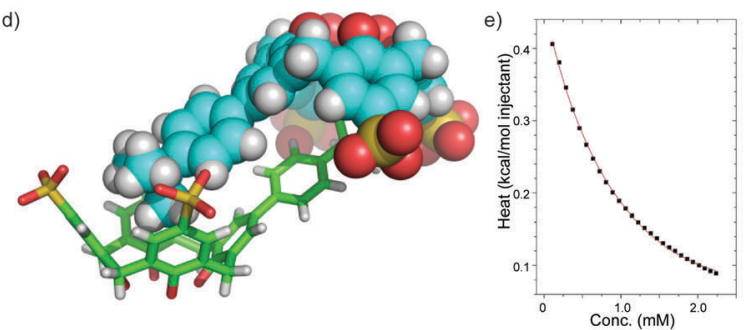

Fig. 1 Well-ordered dimers are formed in solution and the solid state (a) The NMR spectra for compound 2 in phosphate-buffered $\mathrm{D}_{2} \mathrm{O}(100 \mathrm{mM}$ $\mathrm{Na}_{2} \mathrm{HPO}_{4}, \mathrm{pD}$ 7.0) reveal concentration-dependent shifts showing aggregation in water that does not occur in $\mathrm{CD}_{3} \mathrm{OD}$. (b) Compounds with varied $\mathrm{R}$ group all show similar NMR behaviour, suggesting similar assemblies in solution. $\Delta \delta$ values between water (assembled) and methanol (monomeric) for pendant-arm protons in each compound prove an end-on insertion into a calixarene cavity. (c) NOE contacts observed for $\mathbf{5}$ in phosphate buffer. Black arrows indicate NOE's that might have arisen from intramolecular contacts in the monomer, while red arrows can only have arisen from intermolecular self-association consistent with the solid-state structure. See $\mathrm{ESI} \dagger$ for raw NOE data in phosphate buffer and in $\mathrm{CD}_{3} \mathrm{OD}$. (d) The structure of dimeric assemblies is confirmed by the $\mathrm{X}$-ray structure of 5. (e) Exemplary ITC data arising from a dilution of 1 into a matched background buffer, fitted to a dimer dissociation model. See ESI $\dagger$ for all ITC data, and Table 2 for thermodynamic binding data.

of the NMR and ITC experiments. Simple calculations of Coulombic interactions using the partial atomic charges located on adjacent monomers, screened by the dielectric of water, show that the homodimer geometry creates $c a .4 .5 \mathrm{kcal} \mathrm{mol}^{-1}$ of repulsive electrostatic interaction (see ESI $\dagger$ ). Regardless of the real magnitude of that repulsive energy, it is clear that the added salts strengthen the assembly in solution. They might act by increasing the dielectric and screening mutual electrostatic repulsion; they might act by condensing directly onto sulfonates; ${ }^{7}$ they might be increasing the strength of the hydrophobic effect due to 'salting-out' by $\mathrm{NaCl}^{12}$ Whatever the mechanism, the fact that the assembly gets stronger in salt solutions can only be

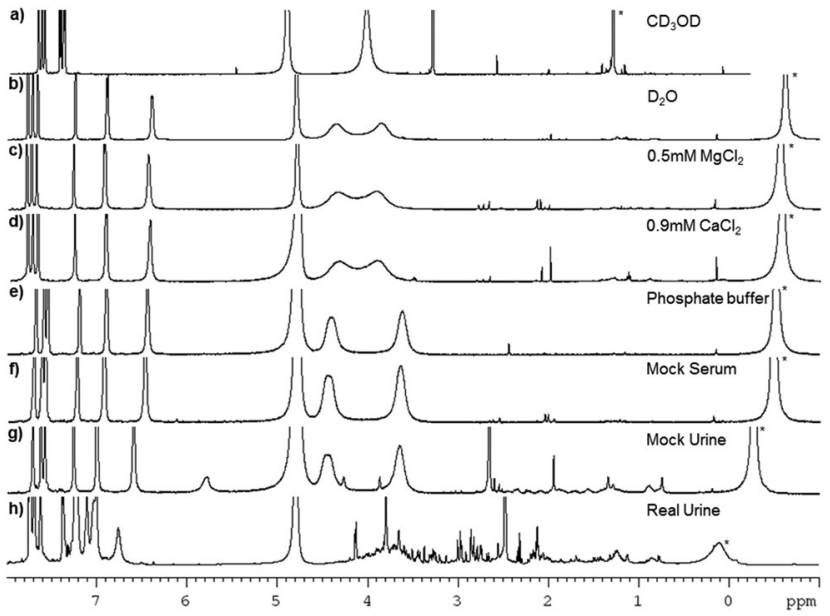

Fig. 2 NMR shows that similar assemblies persist in competitive media. NMR spectra of 5 in: (a) $C D_{3} \mathrm{OD}$ (b) $\mathrm{D}_{2} \mathrm{O}$, (c) $\mathrm{D}_{2} \mathrm{O}+0.5 \mathrm{mM} \mathrm{MgCl}$, (d) $\mathrm{D}_{2} \mathrm{O}+$ $0.9 \mathrm{mM} \mathrm{CaCl}_{2}$, (e) $\mathrm{D}_{2} \mathrm{O}+100 \mathrm{mM} \mathrm{Na} 2 \mathrm{HPO}_{4} \mathrm{pD} 7.0$, (f) mock serum (100 mM $\mathrm{Na}_{2} \mathrm{HPO}_{4} \mathrm{pD} 7.0,138 \mathrm{mM} \mathrm{NaCl}, 2.7 \mathrm{mM} \mathrm{KCl}, 0.5 \mathrm{mM} \mathrm{MgCl} 2,0.9 \mathrm{mM} \mathrm{CaCl}$ ), (g) mock urine (136 mM NaCl, $22.0 \mathrm{mM} \mathrm{KCl}, 15.1 \mathrm{mM} \mathrm{K}_{2} \mathrm{SO}_{4}, 223 \mathrm{mM}$ urea, $13.2 \mathrm{mM}$ creatinine, $0.13 \mathrm{mM}$ corticosterone), (h) real urine $+10 \%(\mathrm{v} / \mathrm{v}) \mathrm{D}_{2} \mathrm{O}$. The key methyl resonance whose shift is diagnostic of assembly is marked with an asterisk.

Table 1 Diffusion constants determined by diffusion ordered spectroscopy (DOSY NMR) support dimerization of $\mathbf{5}$ in solution

\begin{tabular}{llrl}
\hline Compound & $D\left(\mathrm{~m}^{2} \mathrm{~s}^{-1}\right)$ & $r_{\mathrm{H}}(\AA)$ & $\begin{array}{l}\text { Radius from X-ray } \\
\text { structures }(\AA)\end{array}$ \\
\hline PSC (monomer) & \\
Compound 5 (dimer) $^{a}$ & $2.3 \times 10^{-10}$ & 7.5 & $6.6^{b}$ \\
& $1.5 \times 10^{-10}$ & 11.3 & $11.1^{c}$
\end{tabular}

${ }^{a} p$-Sulfonatocalix[4] arene $\left[\right.$ PSC] $=23.2 \mathrm{mM},[5]=21.2 \mathrm{mM}$ in $\mathrm{D}_{2} \mathrm{O}$ ( $\geq 99.9 \%$ D). 2D-DOSY experiments were performed with convection (PFG double stimulated echo) on a $500 \mathrm{MHz}$ NMR. ${ }^{b}$ Measured from longest dimension of calixarene in protein data bank record 3TYI. ${ }^{c}$ Measured from longest dimension using X-ray of homodimer in the current communication.

Table 2 Thermodynamic parameters for assembly in phosphate buffer and phosphate-buffered saline ${ }^{a}$

\begin{tabular}{|c|c|c|c|c|c|c|c|c|}
\hline \multicolumn{3}{|c|}{$K_{\mathrm{d}}(\mathrm{mM})$} & \multicolumn{2}{|c|}{$\underline{\Delta G\left(\mathrm{kcal} \mathrm{mol}^{-1}\right)}$} & \multicolumn{2}{|c|}{$\left.\underline{\Delta H(\mathrm{kcal} \mathrm{mol}}{ }^{-1}\right)$} & \multicolumn{2}{|c|}{$\left.\underline{(-) T \Delta S(\mathrm{kcal} \mathrm{mol}}{ }^{-1}\right)$} \\
\hline & PHOS & PBS & PHOS & PBS & PHOS & PBS & PHOS & PBS \\
\hline 1 & 8.1 & 4.2 & -2.9 & -3.3 & -14.4 & -14.4 & 11.5 & 11.1 \\
\hline 2 & 7.0 & 4.5 & -3.0 & -3.3 & -10.8 & -10.6 & 7.8 & 7.4 \\
\hline 3 & 4.7 & 3.3 & -3.2 & -3.5 & -12.7 & -10.3 & 9.5 & 6.9 \\
\hline 4 & 1.5 & 1.1 & -3.6 & -4.1 & -12.8 & -12.0 & 6.2 & 7.9 \\
\hline 5 & 1.0 & 0.73 & -4.2 & -4.4 & -11.0 & -10.3 & 6.9 & 5.9 \\
\hline 6 & 1.1 & 1.4 & -4.1 & -4.0 & -8.4 & -8.9 & 4.3 & 4.9 \\
\hline
\end{tabular}

${ }^{a}$ Values reported are the average of triplicate ITC dilution titrations. PHOS is $100 \mathrm{mM} \mathrm{NaH} \mathrm{PO}_{4}$ phosphate buffer at $\mathrm{pH} 7.4$, and PBS is the same phosphate buffer containing $\mathrm{NaCl}, \mathrm{KCl}$, and $\mathrm{MgCl}_{2}$ at the levels found in human serum (see ESI). $\mathrm{CaCl}_{2}$ is omitted because of precipitation of calcium phosphates during ITC. All standard deviations for $K_{\mathrm{d}}$ and $\Delta H$ were $\leq 10 \%$ of the reported values. See ESI for all fitted replicates.

attributed to the fact that the assembling monomers, unusually, include mutually repulsive anionic charges.

In order to probe further the influence of the repulsive anionic groups, we prepared compound 6 , in which a cationic $\mathrm{CH}_{2} \mathrm{NH}_{3}{ }^{+}$ 
group takes the place of the alkyl groups in 1-5. Compound 6 shows NMR shifts in water that support assembly as a similar dimer in which the $\mathrm{CH}_{2} \mathrm{NH}_{3}{ }^{+}$group interacts with the pocket and sulfonates of its partner (ESI $\dagger$ ). Some aryl-aryl hydrophobic contacts are retained in the dimer of $\mathbf{6}$, but the mutual electrostatic repulsion is partly replaced with a more conventional cation-anion interaction. Unlike 1-5, ITC shows that the dimer of 6 becomes slightly weaker upon addition of salt, as expected of a dimer that is at least partially driven by electrostatic attraction. Literature examples that involve multiply charged cationic and anionic calix[4]arenes forming heterodimers either precipitate from pure water $^{4}$ or have not been studied in high salt, ${ }^{13,14}$ making direct comparisons between the salt responses of 1-5 and those of purely electrostatically driven dimers difficult.

Self-assembly in nature is a tug-of-war between electrostatics and hydrophobicity. Lipids self-assemble into polymeric aggregates under the control of charged head groups, lipid surface area, and molecular shape parameters. ${ }^{15}$ But the ways that proteins dictate their own discrete assemblies are more subtle and complex. Some have evolved to position charged, repulsive "gatekeeper" residues on aggregation prone surfaces in order to control specificity, maintain solubility, and avoid undesired aggregation-an evolved strategy that has also been called negative design. ${ }^{16,17}$

Compounds 1-5 also use repulsion to temper their mutual hydrophobic attraction, and it occurred to us that this might enable them to remain faithful to each other even in diverse salt conditions and in real biological fluids containing other potential partners for binding and aggregation. Fig. 2 shows the NMR spectra of compound $\mathbf{5}$ in water, water containing added divalent salts, phosphate buffer, mock serum, mock urine, and real human urine. In each case, the characteristic NMR shifts confirm the presence of the assembly. Therefore, these recognition modules can survive the presence of high concentrations of salts, denaturing solutes (urea, creatinine), hydrophobic metabolites (steroids), proteins (albumin), and other dissolved biomolecules. They remain assembled with each other even though other sulfonated calix[4]arenes are known to bind alkali metal ions, ${ }^{7,18}$ steroids, $^{19}$ and albumin. ${ }^{20}$ The more weakly dimerising tolyl monomer 2 shows similar results (ESI $\dagger$ ), showing that robustness in real biological fluids is a general feature of this recognition motif. Few examples exist of host-guest chemistry in untreated urine, a salty and aggressively denaturing medium. ${ }^{21-23}$

The use of like charges in assemblies that persist in high salt has not previously been highlighted as a self-assembly motif, but we identified some recent literature examples that suggest that it is a general route to achieve molecular recognition in salt water. (1) The 'octa-acid' cavitand reported by Gibb bears a $(-8)$ charge, yet binds hydrophobic anions within its interior and shows increased affinity for anion guest binding upon addition of $\mathrm{NaCl}^{24}$ (2) A water-soluble bambusuril host has 12 anionic pendant groups, binds hydrophobic anions in its interior, and retains its anion affinity upon the addition of very high concentrations of salt. ${ }^{25}$ (3) Anionic calixarene hosts, including a sulfonatocalix[8] arene with a net -8 charge, bind and transport the membrane-impermeable, dianionic dye Lucifer yellow across a layer of Caco-2 epithelial cells. ${ }^{26}$ (4) For good measure, a simple tetracationic oxacalixarene host was recently shown to bind to the dicationic guest paraquat in water, although salt effects were not reported. ${ }^{27}$ The motif that is common among these systems is the use of hydrophobic attraction tempered by like-charged electrostatic elements within a rigid, concave molecular host framework.

The emerging picture is that, while approach of like charges is repulsive in the microscopic sense, it can provide net benefits for specific molecular recognition in salty solutions. Where traditional cation + anion designs intrinsically fight against the competing influence of salt, the counterintuitive use of anionic elements to bind other anions is inherently well suited to achieve molecular recognition. Another advantage is that these designs retain excellent solubility in diverse aqueous media, whereas precipitation upon binding and charge neutralization is a common liability for hetero-charged systems of many types. In the face of many results-published and unpublished-of molecular recognition systems that work in polar solvents but fail to survive the move to pure water, added salt and/or dissolution in a real biological fluid, the use of like-charged motifs provides a useful alternative.

We thank Chris Barr for expert assistance with NMR experiments, and David Harrington for electrostatic potential energy calculations. This project was funded by NSERC Discovery Grant 326947. JPD was supported by a scholarship from MITACS. KD was supported by scholarships from WestCoast Ride to Live and Prostate Cancer Foundation of BC. FH is a Canada Research Chair.

\section{Notes and references}

$\ddagger$ Crystallographic data for 5: $\mathrm{C}_{38} \mathrm{H}_{70} \mathrm{Na}_{3} \mathrm{O}_{32} \mathrm{~S}_{3},\left(\mathrm{C}_{38} \mathrm{H}_{32} \mathrm{O}_{13} \mathrm{Na}_{3}\left(\mathrm{H}_{2} \mathrm{O}\right)_{19}\right)$, $M=1204.09,0.14 \times 0.12 \times 0.09 \mathrm{~mm}, T=200 \mathrm{~K}$, hexagonal, space group $P \overline{3} 1 c, a=27.0902(13) \AA, b=27.0902(13) \AA, c=30.1891(16) \AA, V=$ 19186.9(17) $\AA^{3}, Z=12, D_{\mathrm{c}}=1.251 \mathrm{Mg} \mathrm{m}^{-3}, \mu=1.971 \mathrm{~mm}^{-1}, F(000)=$ $7620,2 \theta_{\max }=100.86^{\circ}, 35587$ reflections, 6710 independent reflections $\left[R_{\text {int }}=0.0331\right], R_{1}=0.0794, \mathrm{w} R_{2}=0.2433$ and $\mathrm{GOF}=1.110$ for 6710 reflections (571 parameters) with $I>2 \sigma(I), R_{1}=0.0989, \mathrm{w} R_{2}=0.2670$ and $\mathrm{GOF}=1.110$ for all reflections, $\max / \mathrm{min}$ residual electron density $+0.443 /-0.374$ e $\AA^{3}$. Deposited as CCDC 1033560.

1 R. E. Brewster and S. B. Shuker, J. Am. Chem. Soc., 2002, 124, 7902-7903.

2 T. Grawe, T. Schrader, R. Zadmard and A. Kraft, J. Org. Chem., 2002, 67, 3755-3763.

3 J. Valero and J. de Mendoza, Supramol. Chem., 2013, 25, 728-740.

4 F. Corbellini, R. Fiammengo, P. Timmerman, M. Crego-Calama, K. Versluis, A. J. R. Heck, I. Luyten and D. N. Reinhoudt, J. Am. Chem. Soc., 2002, 124, 6569-6575.

5 G. Hennrich, W. M. David, Y. J. Bomble, E. V. Anslyn, J. S. Brodbelt and J. F. Stanton, Supramol. Chem., 2004, 16, 521-528.

6 M. Rekharsky, Y. Inoue, S. Tobey, A. Metzger and E. Anslyn, J. Am. Chem. Soc., 2002, 124, 14959-14967.

7 V. Francisco, A. Piñeiro, W. M. Nau and L. García-Río, Chem. - Eur. J., 2013, 19, 17809-17820.

8 W. Ong and A. E. Kaifer, J. Org. Chem., 2004, 69, 1383-1385.

9 K. D. Daze, M. C. F. Ma, F. Pineux and F. Hof, Org. Lett., 2012, 14, 1512-1515.

10 S. Tabet, S. F. Douglas, K. D. Daze, G. A. E. Garnett, K. J. H. Allen, E. M. M. Abrioux, T. T. H. Quon, J. E. Wulff and F. Hof, Bioorg. Med. Chem. Lett., 2013, 21, 7004-7010.

11 I. Yoshida, N. Yamamoto, F. Sagara, D. Ishii, K. Ueno and S. Shinkai, Bull. Chem. Soc. Jpn., 1992, 65, 1012-1015.

12 B. C. Gibb, Isr. J. Chem., 2011, 51, 798-806.

13 F. Corbellini, R. M. A. Knegtel, P. D. J. Grootenhuis, M. Crego-Calama and D. N. Reinhoudt, Chem. - Eur. J., 2005, 11, 298-307. 
14 F. Corbellini, L. Di Costanzo, M. Crego-Calama, S. Geremia and D. N. Reinhoudt, J. Am. Chem. Soc., 2003, 125, 9946-9947.

15 V. V. Kumar, Proc. Natl. Acad. Sci. U. S. A., 1991, 88, 444-448.

16 D. E. Otzen, O. Kristensen and M. Oliveberg, Proc. Natl. Acad. Sci. U. S. A., 2000, 97, 9907-9912.

17 R. Sant'Anna, C. Braga, N. Varejão, K. M. Pimenta, R. Graña-Montes, A. Alves, J. Cortines, Y. Cordeiro, S. Ventura and D. Foguel, J. Biol. Chem., 2014, 289, 28324-28337.

18 N. Basilio, V. Francisco and L. Garcia-Rio, Int. J. Mol. Sci., 2013, 14, 3140-3157.

19 J. Millership, J. Inclusion Phenom. Macrocyclic Chem., 2001, 39, 327-331.

20 L. Memmi, A. N. Lazar, A. Brioude, V. Ball and A. W. Coleman, Chem. Commun., 2001, 2474-2475.
21 Y. P. Li, H. Wu and L. M. Du, Chin. Chem. Lett., 2009, 20, $322-325$.

22 E. Biavardi, C. Tudisco, F. Maffei, A. Motta, C. Massera, G. G. Condorelli and E. Dalcanale, Proc. Natl. Acad. Sci. U. S. A., 2012, 109, 2263-2268.

23 D. A. Ryan and J. Rebek, Analyst, 2013, 138, 1008-1010.

24 R. S. Carnegie, C. L. D. Gibb and B. C. Gibb, Angew. Chem., Int. Ed., 2014, 53, 11498-11500.

25 M. A. Yawer, V. Havel and V. Sindelar, Angew. Chem., Int. Ed., 2015, 54, 276-279.

26 E. Roka, M. Vecsernyes, I. Bacskay, C. Felix, M. Rhimi, A. W. Coleman and F. Perret, Chem. Commun., 2015, 51, 9374-9376.

27 N. Manganaro, G. Lando, C. Gargiulli, I. Pisagatti, A. Notti, S. Pappalardo, M. F. Parisi and G. Gattuso, Chem. Commun., 2015, 51, 12657-12660. 